・研究简报・

\title{
青海湖地区狗獾分类地位和狗獾属进化历史探讨
}

\author{
罗 晓 1,2 李 峰 1,3 陈 静 1,2 蒋志刚 $1,2 *$ \\ 1 (中国科学院动物研究所动物生态与保护生物学院重点实验室, 北京 100101) \\ 2 (中国科学院大学, 北京 100049) \\ 3 (江苏大学附属第四人民医院, 江苏镇江 212001)
}

\begin{abstract}
摘要: 本研究选择线粒体细胞色素 $b$ (cytochrome $b$, Cyt $b$ ) 和控制区(control region, CR)片段作为分子标记, 探讨了 青海湖地区狗獾(Meles sp.) 的系统发育地位和狗獾属分歧时间。研究结果支持目前将狗獾属分为 4 个种的结论。Cyt $b$ 和CR片段序列拼接后总长 $1,652 \mathrm{bp}, 23$ 条序列共定义了 21 个单倍型。研究结果表明欧亚大陆狗獾分为东西两个支 系, 每个支系进一步分为两个种: 东部支系包括亚洲狗獾(M. leucurus)和日本狗獾(M. anakuma); 西部支系包括欧 洲狗獾 (M. meles) 和西南亚狗獾( M. canescens)。贝叶斯树和单倍型网络关系图都支持青海湖地区狗獾属于亚洲狗 獾。分歧时间的估算结果与古生物学证据相符, 东部支系和西部支系在 $2.24 \mathrm{Ma}$ 左右产生分歧, 西南亚狗獾在 1.27 $\mathrm{Ma}$ 左右从欧洲狗獾分出，而日本狗獾和亚洲狗獾的分化时间为 $0.99 \mathrm{Ma}$ 左右。
\end{abstract}

关键词: 狗獾; 青海湖地区; 细胞色素 $b$; 控制区; 系统发育关系

\section{The taxonomic status of badgers in the Qinghai Lake area and evolu- tionary history of Meles}

\author{
Xiao Luo ${ }^{1,2}$, Feng $\mathrm{Li}^{1,3}$, Jing Chen ${ }^{1,2}$, Zhigang Jiang ${ }^{1,2^{*}}$ \\ 1 Key Laboratory of Animal Ecology and Conservation Biology, Institute of Zoology, Chinese Academy of Sciences, Bei- \\ jing 100101 \\ 2 University of Chinese Academy of Sciences, Beijing 100049 \\ 3 The 4th Affiliated Hospital of Jiangsu University, Zhenjiang, Jiangsu 212001
}

\begin{abstract}
To investigate the phylogenetic status of badgers (Meles sp.) in the Qinghai Lake area and to estimate the divergence time within Eurasian badgers, the cytochrome $b$ gene and the partial control region (CR) of mitochondrial DNA were examined as genetic markers. Our results indicated that Meles is divided into four species. Twenty one haplotypes were identified in 23 combined sequences (1,652 bp) of Cyt $b$ and CR sequences. Results showed that Eurasian badgers were clearly divided into two major lineages: one of Western lineage, composed of badgers from Europe and Southwest Asia, and the other of Eastern lineage, composed of badgers from North and East Asia (including Japan). Furthermore, the Bayesian tree and the haplotype network indicated that the badgers in the Qinghai Lake area are Asian badgers (M. leucurus). The estimated divergence time was consistent with palaeontological evidence. The first split between the Western lineage and Eastern lineage occurred approximately 2.24 Ma. After the separation, the Southwest Asia clade split from Europe approximately 1.27 Ma and the Japan clade split from continental Asia approximately 0.99 Ma.
\end{abstract}

Key words: badger; Meles; Qinghai Lake area; cytochrome $b$; control region; phylogenetic relationship

狗獾属(Meles)隶属于食肉目鼠科, 是鼠科动物 中分布最广的类群, 现生的狗獾广泛分布于古北界 的森林和草原(图1), 北至斯堪的纳维亚和西伯利 亚，南至伊朗、巴勒斯坦和中国南部，东达日本岛，
西界延伸到伊比利亚半岛(Wozencraft, 2005)。狗獾 起源于上新世亚洲的温带森林, 在晚上新世至早更 新世向西扩散到欧洲(Neal \& Cheeseman, 1996), 在 中更新世地层中发现的狗獾化石的形态与现生狗

收稿日期: 2016-01-03; 接受日期: 2016-05-13

基金项目: 科技基础性专项(2013FY110300)和国家自然科学基金(31372175, 31070348)

* 通讯作者 Author for correspondence. E-mail: jiangzg@ioz.ac.cn 


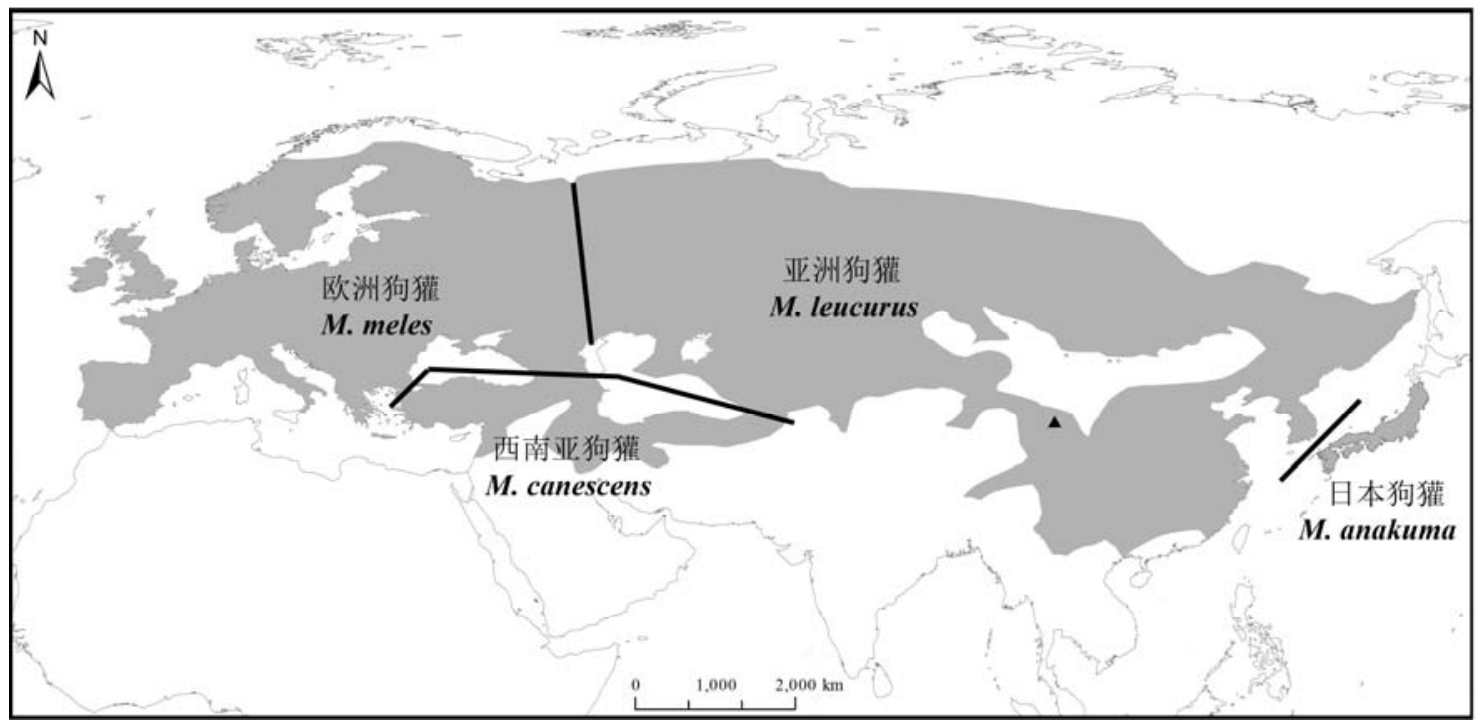

图1 欧亚大陆狗獾分布示意图。灰色区域表示狗獾分布范围, 黑色粗线表示各种群的分界线, 黑色三角符号为采样点。

Fig. 1 Geographic distribution of Eurasian badger. The grey area shows the range of Eurasian badger, the black lines represent geographic boundaries of each phylogenetic group and the black triangle indicates our sampling locality.

獾十分接近(Kurten, 1968; Petter, 1971)。

由于不同地区狗獾形态差异大，狗獾的分类一 直是科学家们争论的话题, 早期研究认为现生狗獾 属仅有1个物种M. meles (Corbet, 1978; Wozencraft, 1993; Nowak \& Paradiso, 1999)。Baryshnikov和 Potapova (1990)基于牙齿形态的差异，将狗獾分为 两个种, 其中欧洲及西南亚地区的狗獾为 M. meles, 而将俄罗斯、中亚和日本的狗獾定为 M. anakuma。 Lynch (1994)依据头骨的大小和形态差异, 认为只 有日本分布的狗獾为 $M$. anakuma, 而其余种群都归 为 M. meles。尔后, Abramov等(Abramov, 2001, 2002; Abramov \& Puzachenko, 2005, 2006)根据阴茎骨和 头骨形态的不同将狗獾分为欧洲狗獾 (M. meles)、亚 洲狗獾(M. leucurus) 和日本狗獾(M. anakuma), 并认 为三者之间的差异达到了种的差异水平。近年来, Abramov和Puzachenko (2013)将欧亚大陆狗獾分为 东部和西部两个支系, 而每个支系又各分为两个不 同的进化支。Marmi等(2006)基于线粒体DNA控制区 序列的分析也将欧亚大陆的狗獾分成了 4 个支系: 即 欧洲狗獾、西南亚狗獾、东亚和北亚狗獾以及日本 狗獾。基于核基因(Del Cerro et al, 2010)和 $S R Y$ 基因 (Tashima et al, 2011a, b) 的分析亦证实了上述结论。

Del Cerro等(2010)以及Abramov和Puzachenko (2013) 提出将西南亚地区分布的狗獾列为独立的种: 高加 索狗獾(M. canescens Blanford, 1875)。但上述系统发
育研究均不包括中国的狗獾样本。

历史上, 狗獾在中国境内广泛分布, 除台湾和 海南省外，中国其余省份均有分布记录(高耀亭, 1987)。但近几十年来由于栖息地破坏和乱捕滥猎, 狗獾数量锐减, 在部分地区已濒临灭绝(谢志刚, 2011; 徐循等, 2012)。尽管狗獾广泛分布于中国, 但 国内对狗獾的研究不多, 且大多集中在食性(叶晓堤 等, 2000; Li et al, 2013)和行为(杨会涛等, 2010; 李峰 和蒋志刚, 2014)。针对狗獾属的系统进化研究中, 关 于中国狗獾的报道只有: Zhou等(2015)测定了1例黑 龙江地区狗獾的线粒体基因组并加入鼠科系统发 育分析, Koh等(2014)将GenBank数据库中中国狗獾 的3 条Cyt $b$ 序列加入亚洲狗獾系统发育分析中。

青海湖地区是已知的狗獾分布区的海拔上限, 为确定青海湖地区狗獾的分类地位, 本研究采集了 狗獾样本，选择线粒体细胞色素 $b(\mathrm{Cyt} b$ ) 和控制区 (control region, CR) 序列作为分子标记, 并从 GenBank数据库中下载其他地区狗獾的序列信息, 对青海湖地区狗獾的系统发育地位进行探讨, 并结 合分歧时间估算来讨论狗獾属的进化历史。

\section{1 材料与方法}

\subsection{1 样本采集}

本实验从青海湖湖东地区采集了 6 只狗獾的组 织样本, 所有样本均用无水乙醇浸泡保存。 


\subsection{2 总DNA提取、目的片段扩增}

取采集的组织样本，按照TIANamp Genomic DNA Kit (天根生化科技有限公司)说明书提取总 DNA。

本研究对细胞色素 $b(\mathrm{Cyt} b)$ 及控制区 $(\mathrm{CR})$ 序列 片段进行扩增。扩增Cyt $b$ 采用Kurose等(2000)设计 的引物Cb-M1 (5'-CTCACATGGAATCTAACCATGAC-3')和Cb-MR1 (5'-TCTTCCTTGAGTCTTAGGG$\left.\mathrm{AG}^{\prime} 3^{\prime}\right)$; 扩增 $\mathrm{CR}$ 采用 Marmi 等(2006) 设计的引物 MelCR1 (5'-AGCACCCAAAGCTGATATTCT-3') 和 MelCR6 (5'-CCATTGACTGAATTGCACCT-3')。

$\mathrm{PCR}$ 反应总体积为 $50 \mu \mathrm{L}$ ，其中 DNA模板 5-10 $\mu \mathrm{L}$, dNTP $1 \mu \mathrm{L}(20 \mathrm{mmol} / \mathrm{L}), 10 \times$ PCR loading buffer $5 \mu \mathrm{L}$, 正反向引物各 $2.5 \mu \mathrm{L}(20 \mathrm{mmol} / \mathrm{L})$, Taq DNA 聚合酶1-2 U, 灭菌 $\mathrm{ddH}_{2} \mathrm{O} 28-33 \mu \mathrm{L}$ 。PCR反应条件 为: $95^{\circ} \mathrm{C}$ 预变性 $5 \mathrm{~min} ; 95^{\circ} \mathrm{C}$ 变性 $45 \mathrm{~s}, 52^{\circ} \mathrm{C}$ 退火 $30 \mathrm{~s}$, $72^{\circ} \mathrm{C}$ 延伸 $45 \mathrm{~s}$, 共 35 个循环; $72^{\circ} \mathrm{C}$ 延伸 $7 \mathrm{~min}, 4^{\circ} \mathrm{C}$ 终
止反应。

\subsection{3 测序和拼接}

PCR产物送北京诺赛基因组研究中心进行测 序，测序引物与扩增引物相同。序列拼接使用 DNASTAR中的SeqMan软件(DNASTAR, Inc.)进行, 测序结果在 $\mathrm{NCBI}$ 数据库中进行比对, 确定采集样 本为狗獾后，使用ClustalX 1.8 (Thompson et al, 2002)进行序列比对。从GenBank数据库中下载17 个样本的Cyt $b$ 和CR序列(表 1 )，由于线粒体基因为 连锁遗传, 为增加系统发育树的准确度, 将Cyt $b$ 和 CR序列在SequenceMatrix (Vaidya et al, 2011)中进 行拼接。

\section{2 数据分析}

\subsection{1 遗传多样性}

使用DnaSP v5.0 (Librado \& Rozas, 2009)进行 遗传多样性检测，计算单倍型数目和核苷酸多样 性。基于Kimura's two-parameter (K2P)模型使用

表1 本研究中的线粒体DNA序列信息

Table 1 The mitochondrial DNA sequences information in this study

\begin{tabular}{|c|c|c|c|c|c|}
\hline \multirow{2}{*}{$\begin{array}{l}\text { 单倍型 } \\
\text { Haplotype }\end{array}$} & \multirow{2}{*}{$\begin{array}{l}\text { 样本代码 } \\
\text { Sample code }\end{array}$} & \multirow{2}{*}{$\begin{array}{l}\text { 样本编号 } \\
\text { Sample no. }\end{array}$} & \multirow{2}{*}{$\begin{array}{l}\text { 采样点 } \\
\text { Sampling locality }\end{array}$} & \multicolumn{2}{|c|}{ 序列号 Accession no. } \\
\hline & & & & Cyt $b$ & Control region \\
\hline H1 & QH1 & B1 & 中国青海 Qinghai, China & KU361236 & KU361238 \\
\hline $\mathrm{H} 2$ & QH2 & B2 & 中国青海 Qinghai, China & KU361237 & KU361239 \\
\hline $\mathrm{H} 2$ & QH3 & B3 & 中国青海 Qinghai, China & KU361237 & KU361239 \\
\hline H3 & QH4 & B4 & 中国青海 Qinghai, China & - & KU361239 \\
\hline H4 & QH5 & B5 & 中国青海 Qinghai, China & - & KU361240 \\
\hline $\mathrm{H} 4$ & QH6 & B6 & 中国青海 Qinghai, China & - & KU361240 \\
\hline H5 & China & - & 中国黑龙江 Heilongjiang, China & KU052604 & KU052604 \\
\hline H6 & Sweden & - & 瑞典 Sweden & AM711900 & AM711900 \\
\hline H7 & Mongolia & $45 \mathrm{Mo}$ & 蒙古 Mongolia & HQ711950 & AJ563694 \\
\hline H8 & Greece & $80 \mathrm{Cr}$ & 希腊克里特岛 Crete Island, Greece & HQ711947 & GU247573 \\
\hline H9 & Israel1 & 49Is & 以色列 Israel & HQ711946 & AJ563686 \\
\hline H10 & Israel2 & $48 \mathrm{Is}$ & 以色列 Israel & HQ711945 & AJ563685 \\
\hline H11 & Spain & $35 \mathrm{Sp}$ & 西班牙 Spain & HQ711943 & AJ563676 \\
\hline H12 & Russia 1 & ZIS33 & 俄罗斯外贝加尔地区 Transbaikalia, Russia & AB049807 & AB538995 \\
\hline H13 & Russia2 & ZIS36 & 俄罗斯列宁格勒州 Leningrad Province, Russia & AB049808 & AB538997 \\
\hline H14 & Russia3 & ZIS35 & 俄罗斯列宁格勒州 Leningrad Province, Russia & AB049809 & AB538998 \\
\hline H15 & Japan1 & K1 & 日本九州大分县 Oita, Kyushu, Japan & AB049806 & AB538971 \\
\hline H16 & Japan2 & K6 & 日本九州大分县 Oita, Kyushu, Japan & AB049800 & AB538971 \\
\hline H17 & Japan3 & K7 & 日本九州大分县 Oita, Kyushu, Japan & AB049802 & AB538971 \\
\hline H18 & Japan4 & K8 & 日本九州福冈县 Fukuoka, Kyushu, Japan & AB049799 & AB538971 \\
\hline H19 & Japan5 & YMG1 & 日本本州山口县 Yamaguchi, Honshu, Japan & AB049795 & AB538983 \\
\hline $\mathrm{H} 20$ & Japan6 & MR1 & 日本九州岩手县 Iwate, Kyushu, Japan & AB049791 & AB538977 \\
\hline \multirow[t]{2}{*}{$\mathrm{H} 21$} & Japan7 & KPM-NF1002945 & 日本本州神奈川县 Kanagawa, Honshu, Japan & AB291075 & AB291075 \\
\hline & Arctonyx collaris & YP6001 & & HM106329 & HM106329 \\
\hline
\end{tabular}


MEGA 6.0软件(Tamura et al，2013)计算种内和种间 遗传差异。

\subsection{2 系统发育分析}

以猪獾 (Arctonyx collaris) 作为外群, 在 MrBayes v3.2 (Ronquist \& Huelsenbeck, 2003)中构 建系统发育树。最佳碱基替换模型在 Modeltest v3.7 软件(Posada \& Crandall, 1998)中进行计算, 选 择赤池信息量准则(Akaike Information Criterion)下 的最佳模型为 $\mathrm{HKY}+\mathrm{I}+\mathrm{G}$ 模型, 程序运行 1,000 万代, 每隔1,000代取样1次, 舍弃(burn in)前 $25 \%$ 的结果。 采用 Network 4.6 (Bandelt et al，1999) 中的 Median-joining法构建单倍型网络图。

\subsection{3 分歧时间估算}

采用BEAST v1.8软件(Drummond \& Rambaut, 2007; Drummond et al, 2012)估算狗獾属分歧时间， 模型设置与贝叶斯分析一致, 分子钟选择 strict clock, 碱基替换速率参照伶鼣(Mustela nivalis)和白 鼠(M. erminea)的 CR碱基替换速率 $1.92 \% /(\mathrm{Ma} \cdot$ site)
(Marmi et al，2006)，采用Song等(2009)中的方法， 计算出联合片段的替换速率为 $2.02 \% /(\mathrm{Ma} \cdot$ site $)$, 程 序运行 1,000 万代，每 1,000 代取样 1 次。在TRACER 1.6 中检验有效性, ESS大于 200 则结果可信。结果采 用TreeAnnotator程序进行总结，并舍弃前 $10 \%$ 的运 行结果。

\section{2 结果}

通过PCR扩增获得青海湖地区狗獾的 3 个Cyt $b$ 全序列和 6 个 CR 序列片段, 并从 GenBank数据库中 下载 17 个样本的Cyt $b$ 和CR片段, 共计 23 个样本。拼 接后线粒体基因全长 $1,652 \mathrm{bp}$, 其中 Cyt $b$ 序列为 $1,140 \mathrm{bp}, \mathrm{CR}$ 序列为 $512 \mathrm{bp}$, 包括 1,373 个保守位点 (conserved sites)、275个可变位点(variable sites)、175 个简约信息位点(parsim-informative sites), 核苷酸 多样性为 $0.026 \pm 0.003$, 共定义 21 个单倍型。

基于线粒体基因构建的贝叶斯系统发育树(图 2)分东部和西部两个大的支系, 其中东部支系又包

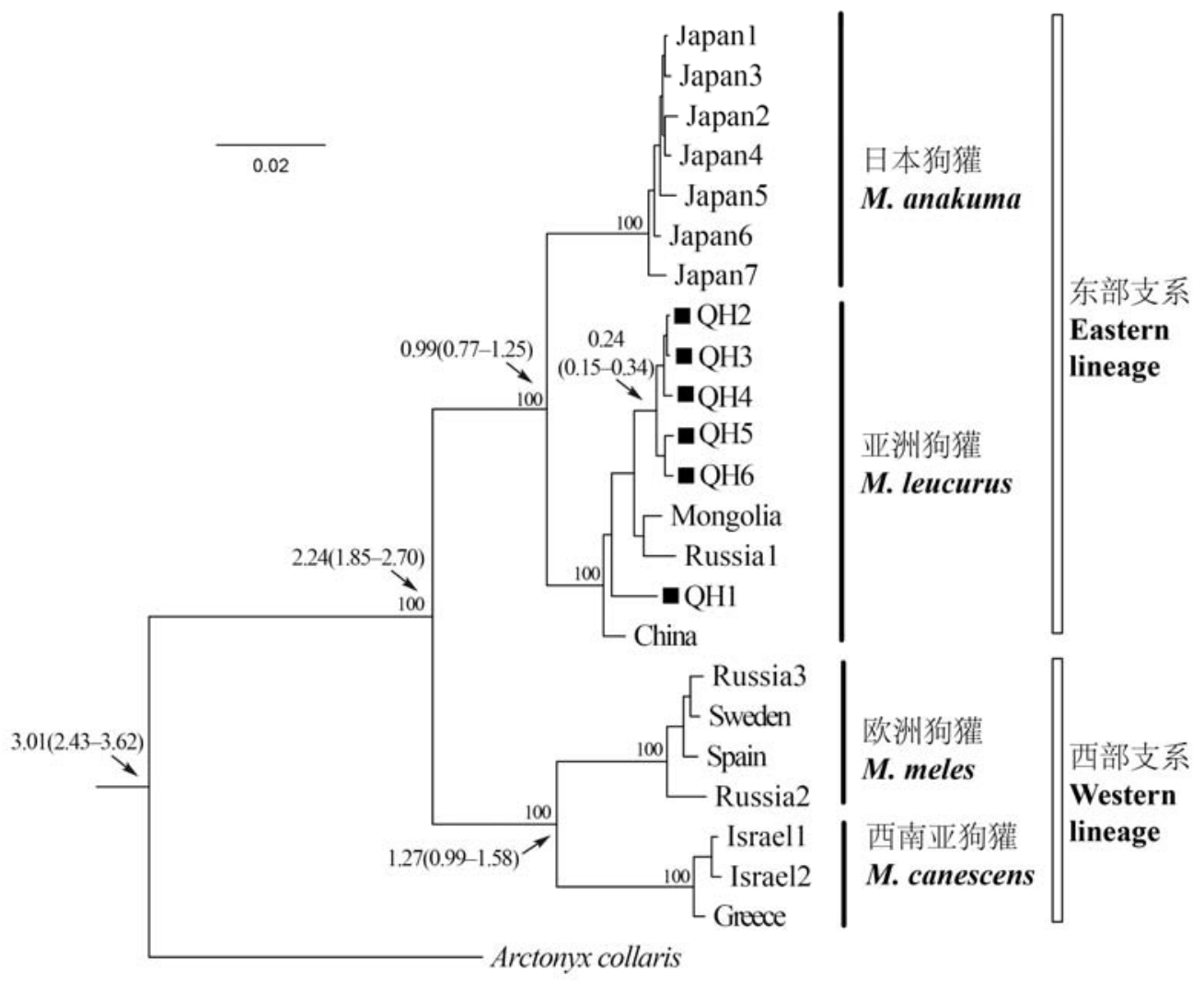

图2 根据线粒体DNA构建的贝叶斯系统发育树。样本代码见表1, 其中QH1-6为青海湖地区狗獾, 枝上数字为主要分支的贝 叶斯后验概率和分歧时间(95\%置信区间)。

Fig. 2 Phylogenetic tree of mitochondrial DNA obtained from Bayesian analysis. The sample codes correspond with those in Table 1, in which QH1-6 represent badgers of Qinghai Lake area. The numbers on the branch indicate Bayesian posterior probability and estimated divergence time (with 95\% HPD). 
表2 欧亚狗獾不同类群间遗传距离

Table 2 Genetic distances among different Eurasian badger groups

\begin{tabular}{lllll}
\hline & $\begin{array}{l}\text { 西南亚狗獾 } \\
\text { M. canescens }\end{array}$ & $\begin{array}{l}\text { 欧洲狗獾 } \\
\text { M. meles }\end{array}$ & $\begin{array}{l}\text { 日本狗獾 } \\
\text { M. anakuma }\end{array}$ & $\begin{array}{l}\text { 亚洲狗獾 } \\
\text { M. leucurus }\end{array}$ \\
\hline 欧洲狗獾 M. meles & $0.034 \pm 0.007$ & & & \\
日本狗獾 M. anakuma & $0.042 \pm 0.009$ & $0.043 \pm 0.008$ & & \\
亚洲狗獾 M. leucurus & $0.040 \pm 0.008$ & $0.041 \pm 0.008$ & $0.021 \pm 0.005$ & $0.017 \pm 0.004$ \\
青海湖地区狗獾 & $0.038 \pm 0.008$ & $0.041 \pm 0.008$ & $0.020 \pm 0.006$ & \\
Badger in the Qinghai Lake area & & & & \\
\hline
\end{tabular}

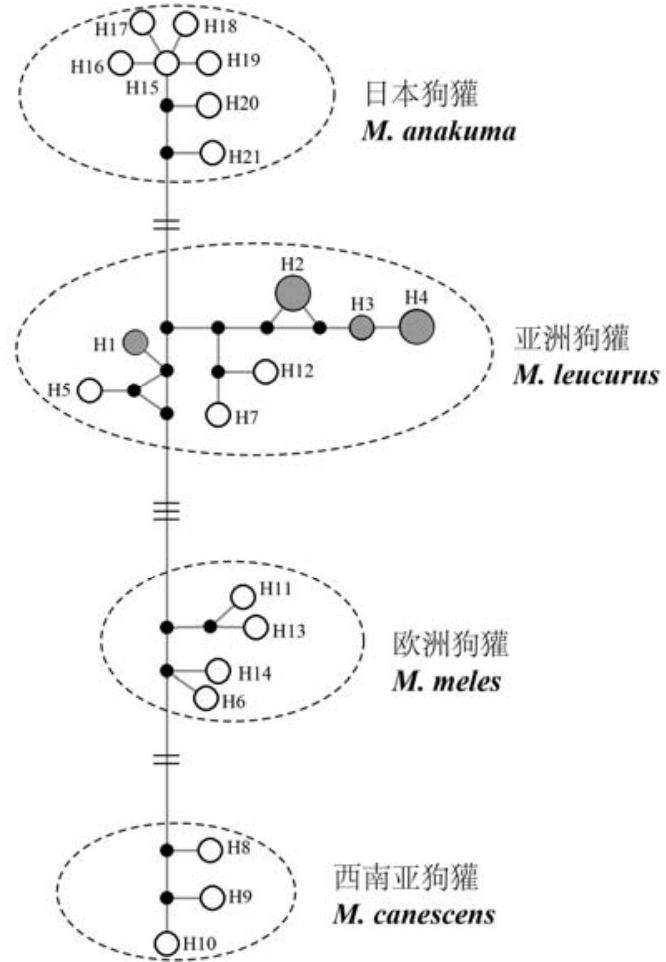

图3 欧亚狗獾线粒体基因 21 个单倍型的网络关系图。单倍 型H1-4为青海湖地区狗獾(灰色), 各单倍型信息见表1, 黑 色圆点表示缺失单倍型, 圆点大小与单倍型频率成正比。对 应的4个分支用虚线圈出。

Fig. 3 The network based on 21 haplotypes of Eurasian badgers mitochondrial DNA. H1-4 represent Qinghai badgers (in grey). All haplotypes are displayed in Table 1 . The black circles represent missing haplotypes. The sizes of the white circles in the network represent the frequencies of each haplotype. Four badger's groups are circled by dotted line.

括亚洲狗獾和日本狗獾两个分支，而西部支系则包 括欧洲狗獾和西南亚狗獾两个分支，每个分支的后 验概率均达到 $100 \%$ 。单倍型网络图(图3)的结构和 系统发育树基本一致, 即欧亚大陆狗獾分为 4 个相 对独立的进化支系, 分别对应欧洲狗獾、亚洲狗獾、 西南亚狗獾和日本狗獾。系统发育树和单倍型网络 关系图中, 青海湖地区狗獾样本与亚洲狗獾聚到
一起。

计算类群间遗传距离时, 我们将青海湖地区的 狗獾作为一个单独的类群处理(表2), 结果显示青海 湖地区狗獾与亚洲狗獾的遗传距离最近, 为 0.017 , 与日本狗獾的遗传距离次之, 而与欧洲狗獾和西南 亚狗獾的遗传距离均较远, 说明青海湖地区狗獾应 该归属于亚洲狗獾。

分歧时间的估算结果在图2的进化树节点处显 示, 狗獾和猪獾在 $3.01 \mathrm{Ma}$ (95\%置信区间：2.43$3.62 \mathrm{Ma}$ )发生分歧, 而东部支系和西部支系的分歧 时间是2.24 Ma (95\%置信区间: 1.85-2.70 Ma)。欧洲 狗獾与西南亚狗獾的分化时间在 $1.27 \mathrm{Ma}(95 \%$ 置信 区间: 0.99-1.58 Ma), 亚洲狗獾和日本狗獾的分化 时间在 $0.99 \mathrm{Ma}$ (95\%置信区间: 0.77-1.25 Ma)。青海 湖地区狗獾QH2-6的分化时间显示为 $0.24 \mathrm{Ma}(95 \%$ 置信区间: 0.15-0.34 Ma)。

\section{3 讨论}

在系统发育树和单倍型网络关系图中, 青海湖 地区的狗獾样本都与亚洲狗獾聚在一起, 并且两者 间的遗传距离最近, 说明青海湖地区的狗獾属于亚 洲狗獾，通过分子遗传学方法确定了青海湖地区狗 獾的分类地位。此外, 在数据分析中, 我们发现青 海湖地区的 $\mathrm{QH} 1$ 与其他几个样本具有较大的遗传 差异, 在系统进化树中并不聚在一起, 从单倍型网 络关系图中可以更清晰地看出 $\mathrm{QH} 1$ 与其他单倍型 相距较远，提示青海湖地区狗獾可能起源于多个最 近祖先, 或者是由多次扩散而来, 也可能是与其他 地区种群有基因交流。

古生物学研究表明狗獾可能起源于上新世时 期中国的Melodon (Viret, 1950), 在晚上新世至早更 新世向西扩散至欧洲(Neal \& Cheeseman, 1996)。本 文对狗獾属分歧时间的估算结果与狗獾的起源和 进化历史基本一致。基于线粒体DNA序列的分析表 
明, 狗獾和猪獾在3.01 Ma (2.43-3.62 Ma)发生分歧, 这与Koepfli等(2008)和Marmi等(2004)的估算结果 基本一致。狗獾的化石种在上新世末期就广泛分布 于古北界(Madurell-Malapeira et al, 2011), 化石证据 表明欧洲狗獾和亚洲狗獾的共同祖先在 $1.8 \mathrm{Ma}$ 前已 发生分歧, 这与本文估算的东西部支系的分歧时间 (2.24 Ma)接近。西南亚狗獾和日本狗獾均受地理隔 离影响而分别与欧洲狗獾、亚洲狗獾发生分歧 (Marmi et al, 2006), 分化时间分别为 $1.27 \mathrm{Ma}(0.99-$ $1.58 \mathrm{Ma})$ 和0.99 Ma (0.77-1.25 Ma), 西南亚狗獾受 到黑海、里海及高加索山脉的阻隔, 而日本狗獾和 亚洲狗獾被日本海峡分隔开来。谱系地理研究认为 欧洲狗獾具有较明显的遗传结构, 推测与末次冰期 狗獾由伊比利亚和欧洲东南部避难所向北扩散有 关(Frantz et al, 2014)。但日本狗獾可能受到末次冰 期后种群扩张的影响, 各种群间并没有明显的分化 (Marmi et al, 2006; Tashima et al, 2011a), 本研究在 青海湖地区采集的 6 个样本没有形成单独的分支, 由于本研究样本有限, 无法对青海湖地区狗獾的系 统分化做出更多解释, 未来需基于更多的中国狗獾 样本对其种群遗传结构进行深入分析。

致谢: 感谢青海湖湖东种羊场牧民花青加、桑杰加 在野外工作时给予的大力帮助, 感谢本研究组李春 旺博士、李忠秋博士、胡军华博士、李春林博士、 以及汤宋华和崔绍朋提供的帮助。

\section{参考文献}

Abramov AV (2001) Notes on the taxonomy of the Siberian badgers (Mustelidae: Meles). Proceedings of the Zoological Institute, Russian Academy of Sciences, 288, 221-233.

Abramov AV (2002) Variation of the baculum structure of the Palaearctic badger (Carnivora, Mustelidae, Meles). Russian Journal of Theriology, 1, 57-60.

Abramov AV, Puzachenko AY (2005) Sexual dimorphism of craniological characters in Eurasian badgers, Meles spp. (Carnivora, Mustelidae). Zoologischer Anzeiger, 244, $11-29$.

Abramov AV, Puzachenko AY (2006) Geographical variability of skull and taxonomy of Eurasian badgers (Mustelidae, Meles). Zoologicheskii Zhurnal, 85, 641-655. (in Russian with English abstract)

Abramov AV, Puzachenko AY (2013) The taxonomic status of badgers (Mammalia, Mustelidae) from Southwest Asia based on cranial morphometrics, with the redescription of Meles canescens. Zootaxa, 3681(1), 044-058.

Bandelt HJ, Forster P, Röhl A (1999) Median-joining networks for inferring intraspecific phylogenies. Molecular Biology and Evolution, 16, 37-48.

Baryshnikov GF, Potapova OR (1990) Variability of the dental system in badgers (Meles, Carnivora) of the USSR fauna. Zoologicheskii Zhurnal, 69, 84-97. (in Russian with English abstract)

Corbet GB (1978) The Mammals of the Palaearctic Region: A Taxonomic Review. Cornell University Press, London and Ithaca.

Del Cerro I, Marmi J, Ferrando A, Chashchin P, Taberlet P, Bosch M (2010) Nuclear and mitochondrial phylogenies provide evidence for four species of Eurasian badgers (Carnivora). Zoologica Scripta, 39, 415-425.

Drummond AJ, Rambaut A (2007) BEAST: Bayesian evolutionary analysis by sampling trees. BMC Evolutionary Biology, 7, 214.

Drummond AJ, Suchard MA, Xie D, Rambaut A (2012) Bayesian phylogenetics with BEAUti and the BEAST 1.7. Molecular Biology and Evolution, 29, 1969-1973.

Frantz AC, McDevitt AD, Pope LC, Kochan J, Davison J, Clements CF, Elmeros M, Molina-Vacas G, Ruiz-Gonzalez A, Balestrieri A, Berge KVD, Breyne P, Do Linh San E, Gren E, Suchentrunk F, Schley L, Kowalczyk R, Kostka BI, Cirovic D, Sprem N, Colyn M, Ghirardi M, Racheva V, Braun C, Oliveira R, Lanszki J, Stubbe A, Stubbe M, Stier N, Burke T (2014) Revisiting the phylogeography and demography of European badgers (Meles meles) based on broad sampling, multiple markers and simulations. Heredity, 113, 443-453.

Gao YT (1987) Fauna Sinica, Mammalia: Vol. 8, Carnivora, pp. 214-223. Science Press, Beijing. (in Chinese) [高耀亭 (1987) 中国动物志·兽纲: 第八卷, 食肉目, 214-223页. 科学出版社, 北京.]

Koepfli KP, Deere KA, Slater GJ, Begg C, Begg K, Grassman L, Lucherini M, Veron G, Wayne RK (2008) Multigene phylogeny of the Mustelidae: resolving relationships, tempo and biogeographic history of a mammalian adaptive radiation. BMC Biology, 6, 1-22.

Koh HS, Kryukov A, Oh JG, Bayarkhagva D, Yang BG, Ahn NH, Bazarsad D (2014) Two sympatric phylogroups of the Asian badger Meles leucurus (Carnivora: Mammalia) identified by mitochondrial DNA cytochrome $b$ gene sequences. Russian Journal of Theriology, 13, 1-8.

Kurose N, Abramov AV, Masuda R (2000) Intrageneric diversity of the cytochrome $b$ gene and phylogeny of Eurasian species of the genus Mustela (Mustelidae, Carnivora). Zoological Science, 17, 673-679.

Kurten B (1968) The Pleistocene Mammals of Europe. Weidenfeld \& Nicolsan, London.

Li F, Jiang ZG (2014) Is nocturnal rhythm of Asian badger (Meles leucurus) caused by human activity? A case study in the eastern area of Qinghai Lake. Biodiversity Science, 22, 758-763. (in Chinese with English abstract) [李峰, 蒋志刚 (2014) 狗獾夜间活动节律是受人类活动影响而形成的 吗? 基于青海湖地区的研究实例. 生物多样性, 22 , 
758-763.]

Li F, Luo ZH, Li CL, Li CW, Jiang ZG (2013) Biogeographical patterns of the diet of Palearctic badger: Is badger an earthworm specialist predator? Chinese Science Bulletin, 58, 2255-2261.

Librado P, Rozas J (2009) DnaSP v5: a software for comprehensive analysis of DNA polymorphism data. Bioinformatics, 25, 1451-1452.

Lynch JM (1994) Morphometric variation in the badger (Meles meles): clinal variation in cranial size and shape across Eurasia. Small Carnivore Conservation, 10, 6-7.

Madurell-Malapeira J, Alba DM, Marmi J, Aurell J, Moyà-Solà S (2011) The taxonomic status of European Plio-Pleistocene badgers. Journal of Vertebrate Paleontology, 31, 885-894.

Marmi J, López-Giráldez F, MacDonald DW, Calafell E, Zholnerovskaya E, Domingo-Roura X (2006) Mitochondrial DNA reveals a strong phylogeographic structure in the badger across Eurasia. Molecular Ecology, 15, 1007-1020.

Marmi J, López-Giráldez JF, Domingo-Roura X (2004) Phylogeny, evolutionary history and taxonomy of the Mustelidae based on sequences of the cytochrome $b$ gene and a complex repetitive flanking region. Zoologica Scripta, 33, 481-499.

Neal E, Cheeseman C (1996) Badgers. T \& AD Poyser Ltd., London.

Nowak RM, Paradiso JL (1999) Walker's Mammals of the World. Cambridge University Press, London.

Petter G (1971) Origine, phylogenie et systematique des blaireaux. Mammalia, 35, 567-597. (in French)

Posada D, Crandall KA (1998) Modeltest: testing the model of DNA substitution. Bioinformatics, 14, 817-818.

Ronquist F, Huelsenbeck JP (2003) MrBayes 3: Bayesian phylogenetic inference under mixed models. Bioinformatics, 19, 1572-1574.

Song G, Qu Y, Yin Z, Li S, Liu N, Lei F (2009) Phylogeography of the Alcippe morrisonia (Aves: Timaliidae): long population history beyond late Pleistocene glaciations. BMC Evolutionary Biology, 9, 143.

Tamura K, Stecher G, Peterson D, Filipski A, Kumar S (2013) MEGA6: molecular evolutionary genetics analysis version 6.0. Molecular Biology and Evolution, 30, 2725-2729.

Tashima S, Kaneko Y, Anezaki T, Baba M, Yachimori S, Abramov AV, Saveljev AP, Masuda R (2011a) Phylogeographic sympatry and isolation of the Eurasian badgers (Meles, Mustelidae, Carnivora): implications for an alternative analysis using maternally as well as paternally inherited genes. Zoological Science, 28, 293-303.

Tashima S, Kaneko Y, Anezaki T, Baba M, Yachimori S, Abramov AV, Saveljev AP, Masuda R (2011b) Identification and molecular variations of CAN-SINEs from the ZFY gene final intron of the Eurasian badgers (genus Meles). Mammal Study, 36, 41-48.
Thompson JD, Gibson T, Higgins DG (2002) Multiple sequence alignment using ClustalW and ClustalX. Current Protocols in Bioinformatics, Chapter 2 (Unit 2.3), doi: 10.1002/0471250953.bi0203s00.

Vaidya G, Lohman DJ, Meier R (2011) SequenceMatrix: concatenation software for the fast assembly of multi-gene datasets with character set and codon information. Cladistics, 27, 171-180.

Viret J (1950) Meles thorali n. sp. du loess villafranchien de Saint-Vallier (Drôme). Eclogae Geologicae Helvetiae, 43, 274-287. (in French with English abstract)

Wozencraft WC (1993) Order Carnivora. In: Mammal Species of the World: A Taxonomic and Geographic Reference, 2nd edn. (eds Wilson DE, Reeder DM), pp. 279-348. Smithsonian Institution Press, Washington, DC.

Wozencraft WC (2005) Order Carnivora. In: Mammal Species of the World: A Taxonomic and Geographic Reference, 3rd edn. (eds Wilson DE, Reeder DM), pp. 532-628. Johns Hopkins University Press, Baltimore.

Xie ZG (2011) Studies on the Population Ecology of Badger (Meles meles) in Shanghai. PhD dissertation, East China Normal University, Shanghai. (in Chinese with English abstract) [谢志刚 (2011) 上海地区狗獾生态学研究. 博士 学位论文, 华东师范大学, 上海.]

Xu X, Xie ZG, Cui YY, Chu KL, Jiang WZ, Pei EL, Xu HF (2012) Activity patterns of reintroduced badgers in seminatural area. Chinese Journal of Zoology, 47(3), 49-52. (in Chinese with English abstract) [徐循, 谢志刚, 崔勇勇, 褚 可龙, 蒋文忠, 裴恩乐, 徐宏发 (2012) 重引入狗獾秋冬 季行为的初步研究. 动物学杂志, 47(3), 49-52.]

Yang HT, Liu ZS, Xu K, Song CL, Wu MF, Sun JH (2010) Autumn habitat selection of Eurasian badgers (Meles meles amurensis): a case of Fangzheng Forestry Bureau, Heilongjiang Province, China. Acta Ecologica Sinica, 30, 1875-1881. (in Chinese with English abstract) [杨会涛, 刘 振生, 徐坤, 宋丛亮, 吴木芬, 孙景海 (2010) 狗獾秋季 对生境的选择一以黑龙江省方正林业局为例. 生态学 报, 30, 1875-1881.]

Ye XD, Ma Y, Wang RH, Dong AY (2000) Review of the diet of Eurasian badgers. Chinese Journal of Zoology, 35(2), 43-50. (in Chinese) [叶晓堤, 马勇, 王润海, 董安渝 (2000) 欧亚大陆狗獾食性的研究概述. 动物学杂志, 35(2), 43-50.]

Zhou WW, Yu L, Tan BY, Liu YT, Zhang L, Hua Y (2015) Phylogenetic relationship of Asian badger Meles leucurus amurensis revealed by complete mitochondrial genome. Mitochondrial DNA, published online, doi: 10.3109/ 19401736.2015.1127365.

(责任编委: 蒋学龙 责任编辑: 闵文杰) 